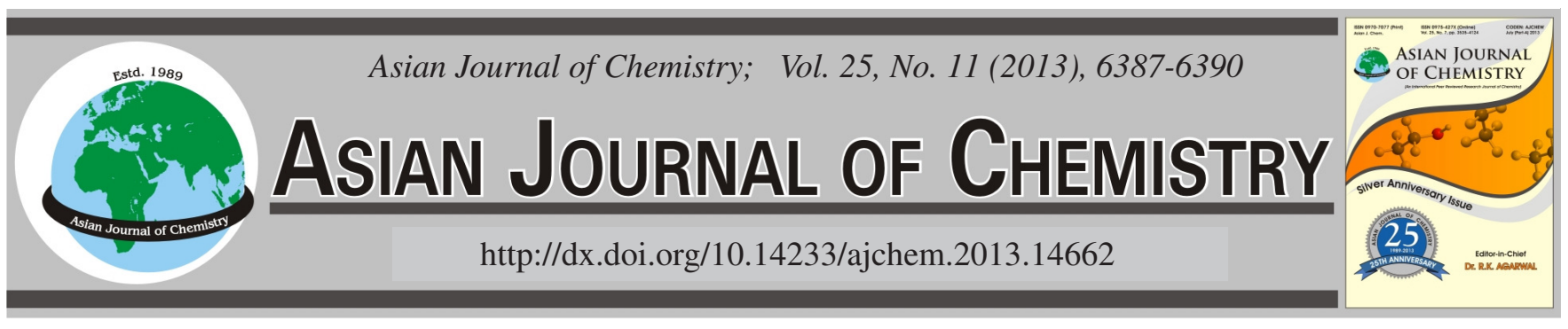

\title{
An Efficient Synthesis of Dichotomine A via Cyclization of L-Tryptophan Derivative
}

\author{
XIU XIAO ShI ${ }^{1}$, Dian HE ${ }^{1, *}$, Shao BaI LI $I^{2}$, XIN Lei ${ }^{2}$ and Huan YANG $^{1}$
}

${ }^{1}$ School of Pharmacy, Lanzhou University, Lanzhou 730000, P.R. China

${ }^{2}$ State Key Laboratory of Applied Organic Chemistry, Lanzhou University, Lanzhou 730000, P.R. China

*Corresponding author: Fax: +86 931 8915686; Tel: +86 13008738922; E-mail: hed@1zu.edu.cn

(Received: 11 October 2012;

Accepted: 10 May 2013)

AJC-13477

A new synthetic method for dichotomine A is reported. The key step of the synthetic process is the efficient cyclization of L-tryptophan derivative via a modified Bischler-Napieralski reaction which aluminium chloride is used as a mild catalyst. Dichotomine A is obtained in 5 steps and $63.9 \%$ yield.

Key Words: Dichotomine A, $\beta$-Carboline alkaloids, Aluminium chloride, Cyclization.

L - - - -

\section{INTRODUCTION}

$\beta$-Carbolines represent a large group of alkaloids widely distributed in nature, occurring in plants, marine organisms and insects, most importantly, in foods and in human tissues and body fluids. They have diverse medicinal properties ${ }^{1-6}$ such as inhibition of topoisomerase and monoamine oxidase, binding to benzodiazepine and serotonin receptors and intercalating into DNA. Dichotomines A, B, C and D, new $\beta$-carboline-type alkaloids, were first isolated from the roots of Stellaria dichotoma in 2004 ${ }^{7}$. They showed an antiallergic effect on ear passive cutaneous anaphylaxis reaction in mice and inhibitory activity on the release of $\beta$-hexosaminidase in RBL-2H3 cells. As shown in Fig. 1, the structure of dichotomines A, B, C and $\mathrm{D}$ include a chiral group respectively. Although many methodologies concerned the synthesis of $\beta$-carbolines ${ }^{8-13}$, the synthesis of dichotomines is quite limited. Dichotomine $\mathrm{C}$ was obtained by Omura and co-workers ${ }^{14}$ based on the microwave assisted thermal electrocyclic reaction of a 1-azahexatriene system. Nemet and Defterdarovic ${ }^{15}$ had synthesized dichotomine A as racemic mixture via reactions of L-tryptophan or L-tryptophan methyl ester with methyl glyoxal under acidic conditions. Zhang and co-works ${ }^{16}$ had synthesized dichotomines A, B, C and D starting from L-tryptophan methyl ester and 2,3-Oisopropylidene-D-glyceraldehyde. From this total synthesis, dichotomine A was obtained in 13 steps and only in $24 \%$ yield. So, we developed a new synthetic method for dichotomine A, which could reduce steps and obtain a higher yield.

We chose L-tryptophan and L-lactic acid as raw material because they were inexpensive, commercially available and the potentially same chiral group of dichotomine A. The synthesis route was designed in Scheme-I.

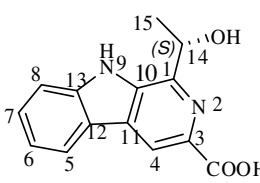

Dichotomine A

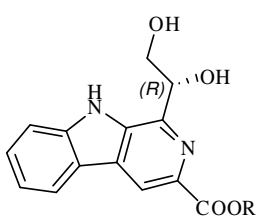

Dichotomine B: $\mathrm{R}=\mathrm{H}$ Dichotomine C: $\mathrm{R}=\mathrm{CH}_{3} \mathrm{CH}_{2} \mathrm{CH}_{3}$

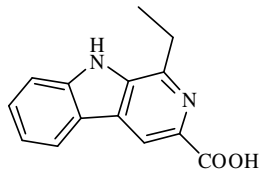

Compound A
Fig. 1. Structure of dichotomines

\section{EXPERIMENTAL}

Melting point was determined in capillary tubes on an electrothermal PIF YRT-3 apparatus and without correction. The optical rotation of dichotomine A was measured with a WZZ-1S polarimeter at room temperature. All reactions were monitored by analytical thin-layer chromatography and silica gel $F_{254}$ was used in TLC. Merck kieselgel 60 (230-400 mesh) was used for column chromatography and ethyl acetate/ petroleum ether mixtures served as the mobile phase. The structure of all compounds was confirmed by ${ }^{1} \mathrm{H}$ NMR, ${ }^{13} \mathrm{C}$ NMR spectra and mass spectroscopy. The ${ }^{1} \mathrm{H}$ NMR and ${ }^{13} \mathrm{C}$ NMR spectra were obtained on Bruker Avance-300 NMR spectrometers in $\mathrm{CDCl}_{3}$ with tetramethylsilane (TMS) as an internal standard, unless otherwise stated. Chemical shifts were reported as $\delta(\mathrm{ppm})$. Mass Spectra was measured on a HP-5988 mass spectrometer and optical rotation was detected by WXG-4 polarimeters.

Synthesis of (S)-2-acetoxypropionyl chloride (10): The mixture of L-lactic acid (7) $(20 \mathrm{mmol})$ and acetic anhydride 
<smiles>C[As](O)C(=O)O</smiles>

7 L-lactic acid<smiles></smiles>

8<smiles>C[14C](=O)[14C](=O)O</smiles>

9<smiles></smiles>

b<smiles></smiles>

10<smiles>CO[14C](N)Cc1c[nH]c2cc(CC(N)[C@@H](N)Cc3c[nH]c4ccccc34)ccc12</smiles>

L-trytophan
2<smiles>COC(=O)N[C@@H](Cc1c[nH]c2ccccc12)C(=O)OC</smiles>

3

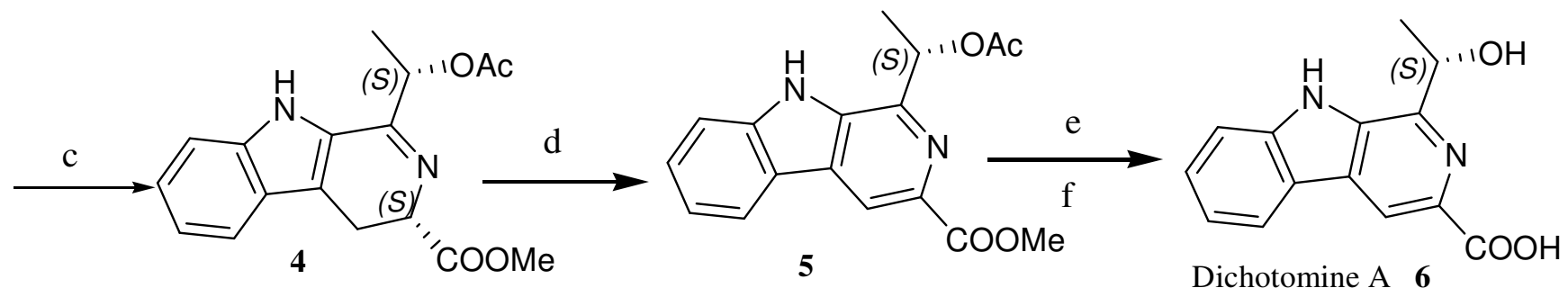

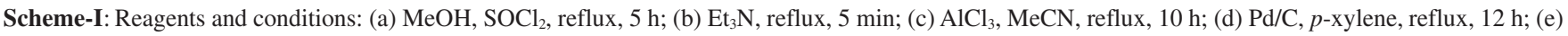
$\mathrm{NaOH} / \mathrm{H}_{2} \mathrm{O}$, EtOH, reflux, 1h; (f) $\mathrm{HCl}$; (g) acetic anhydride, 4 h; (h) $\mathrm{HCl} / \mathrm{H}_{2} \mathrm{O}, 15$ min; (i) $\mathrm{SOCl}_{2}, \mathrm{CH}_{2} \mathrm{ClCH}_{2} \mathrm{Cl}$, reflux, 6 h

(6 mL, $6.37 \mathrm{~g}, 62 \mathrm{mmol})$ stirred for $4 \mathrm{~h}$ at $80^{\circ} \mathrm{C}$ led to compound (8) and was cooled to $40^{\circ} \mathrm{C}$, then the solution of water $(1.2 \mathrm{~mL})$ and $\mathrm{HCl}(0.1 \mathrm{~mL})$ was added quickly and after being stirred for $15 \mathrm{~min}$ at ambient temperature, 1,2-dichloroethane $(40 \mathrm{~mL})$ and thionyl chloride $(10 \mathrm{~mL})$ were also added. The mixture was stirred for $3 \mathrm{~h}$ at $40^{\circ} \mathrm{C}$ and refluxed for $4 \mathrm{~h}$. Then the byproduct acetyl chloride translated from the relic acetic acid and thionyl chloride was distilled off at low pressure. The obtained crude product was not purified, used directly.

Preparation of L-tryptophan methyl ester (2): Thionyl chloride was added to the solution of L-tryptophan (1) (0.36 $\mathrm{g}, 1.8 \mathrm{mmol})$ in excess anhydrous methanol $(10 \mathrm{~mL})$ at $0{ }^{\circ} \mathrm{C}$. The mixture was allowed to warm to ambient temperature and then stirred for $5 \mathrm{~h}$ at reflux temperature. The methanol was evaporated under reduced pressure and the residue was alkalized by $10 \%$ sodium bicarbonate solution until the $\mathrm{pH}$ value was adjusted to 8-9. The solution was extracted with 1,2dichloroethane $(3 \times 10 \mathrm{~mL})$ and the organic phase was dried over anhydrous sodium sulfate and concentrated under reduced pressure affording compound $\mathbf{2}(0.39 \mathrm{~g}, 98 \%)$ as colourless oil:

MS m/z $218\left(\mathrm{M}^{+}, 46\right), 130$ (100), 159 (58); ${ }^{1} \mathrm{H}$ NMR (300 $\left.\mathrm{MHz} \mathrm{CDCl}_{3}\right) \delta 8.76(1 \mathrm{H}, \mathrm{s}, \mathrm{NH}), 7.61(1 \mathrm{H}, \mathrm{d}, J=8.0 \mathrm{~Hz})$, $7.32(1 \mathrm{H}, \mathrm{d}, J=7.9 \mathrm{~Hz}), 7.18(1 \mathrm{H}, \mathrm{t}, J=8.2 \mathrm{~Hz}), 7.01(1 \mathrm{H}, \mathrm{t}$, $J=8.1 \mathrm{~Hz}), 6.97(1 \mathrm{H}, \mathrm{s}, \mathrm{H}-2), 3.86(1 \mathrm{H}, \mathrm{m}, 11-\mathrm{H}), 3.71(3 \mathrm{H}$, s, $\left.-\mathrm{OCH}_{3}\right), 3.29\left(1 \mathrm{H}, \mathrm{dd}, J=4.8,4.3 \mathrm{~Hz}, \mathrm{CH}_{2}\right), 3.06(1 \mathrm{H}, \mathrm{dd}$, $\left.J=6.6,6.1 \mathrm{~Hz}, \mathrm{CH}_{2}\right), 1.69\left(2 \mathrm{H}, \mathrm{s},-\mathrm{NH}_{2}\right) ;{ }^{13} \mathrm{C}$ NMR $(75 \mathrm{MHz}$ $\left.\mathrm{CDCl}_{3}\right): \delta 30.6\left(\mathrm{CH}_{2}, \mathrm{C}-10\right), 51.9\left(-\mathrm{CH}_{3}, \mathrm{C}-13\right), 54.8(-\mathrm{CH}, \mathrm{C}-$ 11), 110.5 (CH, C-3), 111.2 (CH, C-8), 118.5 (CH, C-5), 119.2 (CH, C-7), 121.9 (CH, C-6), 123.1 (CH, C-2), 127.3 (C, C-4), 136.2 (C, C-9), 175.6 (CO, C-12).

Synthesis of $\mathrm{N}$-(O-acetoxypropionyl)-L-tryptophan methyl ester (3): Triethylamine $0.25 \mathrm{~mL}(5.4 \mathrm{mmol})$ was added to the stirring solution of L-tryptophan methyl ester (2) $0.39 \mathrm{~g}(1.8 \mathrm{mmol})$ in $10 \mathrm{~mL}$ dry 1, 2-dichloroethane. At a low temperature, (S)-2-acetoxypropionyl chloride (10) was added dropwise and the mixture was monitored by TLC (ethyl acetate/ petroleum ether $=1: 1$ ) until the complete disappearance of L-tryptophan methyl ester on TLC. The solution was washed with saturated salt water $(3 \times 10 \mathrm{~mL})$. 1,2-dichloroethane was steamed out under the reduced pressure. The residue was purified by column chromatography using petroleum ether/ ethyl acetate $(2: 1, \mathrm{v} / \mathrm{v})$ as the eluent to give $\mathrm{N}-(\mathrm{O}$-acetoxypropionyl)-L-tryptophan methyl ester (3) $(0.39 \mathrm{~g}, 88.6 \%)$ as colourless oil:

MS m/z $332\left(\mathrm{M}^{+}, 10\right), 272$ (12), 130 (100), 201 (38); ${ }^{1} \mathrm{H}$ $\operatorname{NMR}\left(300 \mathrm{MHz} \mathrm{CDCl}_{3}\right) \delta 8.25(1 \mathrm{H}, \mathrm{s}, \mathrm{NH}), 7.53(1 \mathrm{H}, \mathrm{d}, J=$ $8.0 \mathrm{~Hz}, \mathrm{NHCO}), 7.36(1 \mathrm{H}, \mathrm{d}, J=7.9 \mathrm{~Hz}, \mathrm{H}-5), 7.14(2 \mathrm{H}, \mathrm{m}$, H-6, 7), $7.01(1 \mathrm{H}, \mathrm{s}, \mathrm{H}-8), 6.53(1 \mathrm{H}, \mathrm{s}, \mathrm{CH}), 4.93(1 \mathrm{H}, \mathrm{m}, \mathrm{H}-$ 11), $4.51(1 \mathrm{H}, \mathrm{m}, \mathrm{H}-14), 3.35\left(3 \mathrm{H}, \mathrm{s},-\mathrm{OCH}_{3}\right), 3.29(1 \mathrm{H}, \mathrm{dd}, J$ $\left.=4.9,12.4 \mathrm{~Hz}, \mathrm{H}-10^{\prime}\right), 2.84\left(1 \mathrm{H}, \mathrm{dd}, J=6.8,12.8 \mathrm{~Hz}, \mathrm{H}-10^{\prime \prime}\right)$, $1.94(3 \mathrm{H}, \mathrm{s},-\mathrm{OAc}), 1.38\left(3 \mathrm{H}, \mathrm{m},-\mathrm{CH}_{3}\right) ;{ }^{13} \mathrm{C}$ NMR $(75 \mathrm{MHz}$ $\left.\mathrm{CDCl}_{3}\right): \delta 16.2\left(\mathrm{CH}_{3}, \mathrm{C}-15\right), 20.3\left(\mathrm{CH}_{3}, \mathrm{C}-17\right), 30.9\left(\mathrm{CH}_{2}, \mathrm{C}-\right.$ 10), $51.6\left(\mathrm{CH}_{3}, \mathrm{C}-19\right), 54.8$ (CH, C-11), $79.5(\mathrm{CH}, \mathrm{C}-14), 110.9$ (C, C-3), 111.2 (CH, C-8), 119.1 (CH, C-5), 120.3 (CH, C-7), 122.6 (CH, C-6), 122.4 (CH, C-2), 127.2 (C, C-4), 136.5 (C, C-9), 170.3 (C, C-18), 171.6 ( C, C-16), 172.4 (C, C-13).

Synthesis of 1-(1'-O-acetoxypropionyl)-ethyl-3-(methoxycarbonyl)-(3, 4-2H)- $\beta$-carboline (4): To a solution of compound $\mathbf{3}(1.0 \mathrm{~g}, 3 \mathrm{mmol})$ in anhydrous acetonitrile $(30 \mathrm{~mL})$, aluminium chloride $(0.1 \mathrm{~g}$, 1equiv) was added. After the mixture was stirred for $10 \mathrm{~h}$ at $80^{\circ} \mathrm{C}$, the catalyst was removed by filtration and the solution was distilled off under reduced pressure. The caput mortuum was dissolved in dichloromethane $(30 \mathrm{~mL})$ and washed with water $(3 \times 20 \mathrm{~mL})$. The dichloro- 
methane was dried over anhydrous sodium sulfate and distilled off under reduced pressure. The residue was purified by column chromatography using petroleum ether/ethyl acetate $(5: 3, \mathrm{v} / \mathrm{v})$ as the eluent to give the compound $4(0.82 \mathrm{~g}$, $87.3 \%)$ as colourless oil:

MS m/z $314\left(\mathrm{M}^{+}, 12\right), 255$ (63), 196 (100), 168 (45), 147 (85), 122 (56), 59 (65); ${ }^{1} \mathrm{H}$ NMR (300 MHz $\left.\mathrm{CDCl}_{3}\right) \delta 8.25$ (1H, s, NH-9), 7.53 (1H, d, $J=8.0 \mathrm{~Hz}, \mathrm{H}-5), 7.36(1 \mathrm{H}, \mathrm{d}, J=$ 7.8Hz, H-8), 7.14 (2H, m, H-6, 7), 4.93 (1H, m, H-14), 4.51 $(1 \mathrm{H}, \mathrm{m}, \mathrm{H}-3), 3.65\left(3 \mathrm{H}, \mathrm{s},-\mathrm{OCH}_{3}\right), 3.29(1 \mathrm{H}, \mathrm{dd}, J=4.6,12.8$ Hz, H-4'), 2.88 (1H, dd, J=6.9, 12.4 Hz, H-4"), 1.94 (3H, s, $\mathrm{H}-18), 1.38(3 \mathrm{H}, \mathrm{d}, J=5.8 \mathrm{~Hz}, \mathrm{H}-15) ;{ }^{13} \mathrm{C} \mathrm{NMR}(75 \mathrm{MHz}$ $\left.\mathrm{CDCl}_{3}\right): \delta 14.1\left(\mathrm{CH}_{3}, \mathrm{C}-15\right), 20.7\left(\mathrm{CH}_{3}, \mathrm{C}-19\right), 30.3\left(\mathrm{CH}_{2}\right.$, $\mathrm{C}-4), 51.9\left(\mathrm{CH}_{3}, \mathrm{C}-17\right), 64.9(\mathrm{CH}, \mathrm{C}-3), 75.5(\mathrm{CH}, \mathrm{C}-14), 111.1$ $(\mathrm{CH}, \mathrm{C}-8), 112.9(\mathrm{CH}, \mathrm{C}-5), 118.8(\mathrm{CH}, \mathrm{C}-7), 119.1(\mathrm{CH}$, C-6), 121.7 (C, C-12), 125.9 (C, C-11), 126.6 (C, C-10), 138.7 (C, C-13), 170.3 (C, C-18), 173.4 (C, C-16).

Synthesis of 1-(1'-O-acetoxypropionyl)-ethyl-3(meth-oxycarbonyl)- $\boldsymbol{\beta}$-carboline (5): Compound 4 (1 mmol) was dissolved in $p$-xylene $(10 \mathrm{~mL})$ and $5 \% \mathrm{Pd} / \mathrm{C}(0.01 \mathrm{~g})$ was added. The mixture was refluxed for $12 \mathrm{~h}$, then the catalyst was removed by filtration and $p$-xylene was distilled off under reduced pressure. The residue was dissolved in chloroform and the solution was washed with ammonium hydroxide. Then the organic layer was separated and the aqueous layer was washed several times with chloroform. All organic layers were combined and dried by $\mathrm{K}_{2} \mathrm{CO}_{3}$. After distilling off the chloroform under reduced pressure, the remained oil was chromatographed on petroleum ether/ethyl acetate $(5: 3, \mathrm{v} / \mathrm{v})$ to provide yellow oil compound 5 (86.0\% yield).

MS m/z $312\left(\mathrm{M}^{+}, 8\right), 254$ (82), 194 (100), 167 (54), 149 (86), 120 (60), 59 (56); ${ }^{1} \mathrm{H}$ NMR (300 MHz CDCl $) \delta 10.61$ (1H, s, NH-9), 8.39 (1H, s, H-4), $8.12(1 \mathrm{H}, \mathrm{d}, J=7.5 \mathrm{~Hz}, \mathrm{H}-$ 5), $7.63(1 \mathrm{H}, \mathrm{d}, J=7.6 \mathrm{~Hz}, \mathrm{H}-8), 7.50(1 \mathrm{H}, \mathrm{t}, J=7.8 \mathrm{~Hz}, \mathrm{H}-7)$, 7.29 (1H, t $J=7.9 \mathrm{~Hz}, \mathrm{H}-6), 5.42(1 \mathrm{H}, \mathrm{m}, \mathrm{H}-14), 3.89$ (3H, s, $\left.-\mathrm{OCH}_{3}\right), 2.21$ (3H, s, H-18), $1.28(3 \mathrm{H}, \mathrm{d}, J=5.4 \mathrm{~Hz}, \mathrm{H}-15)$; ${ }^{13} \mathrm{C} \mathrm{NMR}\left(75 \mathrm{MHz} \mathrm{CDCl}_{3}\right): \delta 19.6\left(\mathrm{CH}_{3}, \mathrm{C}-15\right), 21.1\left(\mathrm{CH}_{3}, \mathrm{C}-\right.$ 19), $51.6\left(\mathrm{CH}_{3}, \mathrm{C}-17\right), 71.1(\mathrm{CH}, \mathrm{C}-14), 111.2(\mathrm{CH}, \mathrm{C}-8), 113.4$ $(\mathrm{CH}, \mathrm{C}-4), 119.1$ (CH, C-5), $119.6(\mathrm{CH}, \mathrm{C}-7), 121.3(\mathrm{CH}, \mathrm{C}-$ 6), 121.6 (C, C-11), 127.4 (C, C-12), 131.0 (C, C-10), 133.6 (C, C-1), 143.2 (C, C-13), 148.5 (C, C-3), 165.3 (C, C-16), 170.3 (C, C-18).

Synthesis of dichotomine A (6): To the solution of compound 5 (1.25 g, $4 \mathrm{mmol})$ in ethanol solvent (10 mL), NaOH/ $\mathrm{H}_{2} \mathrm{O}(12 \mathrm{mmol} / 2 \mathrm{~mL})$ was added. The mixture was refluxed for $2 \mathrm{~h}$ and then the solution was extracted with a mixture of ethyl acetate and water $(1: 1, \mathrm{v} / \mathrm{v})$. The aqueous phase was separated and then acidified with aqueous $\mathrm{HCl}(20 \mathrm{mmol})$. Dichotomine A was crystallized as yellowish powder (1.0 g, $98.0 \%$ yield). Dichotomine A: m.p. 267.1-267.5 ${ }^{\circ} \mathrm{C}$, [a]20D $9.1^{\circ}(\mathrm{C}=0.8, \mathrm{MeOH})$.

MS m/z $256\left(\mathrm{M}^{+}, 10\right), 238$ (20), 223 (68), 149 (100); ${ }^{1} \mathrm{H}$ NMR (400 MHz DMSO-d $), \delta$ (ppm) 10.82 (1H, s, NH-9), $7.65(1 \mathrm{H}, \mathrm{d}, J=7.8 \mathrm{~Hz}, \mathrm{H}-4), 7.35$ (2H, d, $J=7.5 \mathrm{~Hz}, \mathrm{H}-5,8)$, 7.27 (1H, m, H-7), 7.02 (1H, m, H-6), 5.78 (1H, m, H-14), $1.66(1 \mathrm{H}, \mathrm{s},-\mathrm{OH}), 1.18(3 \mathrm{H}, \mathrm{d}, J=7.4 \mathrm{~Hz}, \mathrm{H}-15) ;{ }^{13} \mathrm{C} \mathrm{NMR}$ $\left(100 \mathrm{MHz}\right.$ DMSO- $\left.d_{6}\right) \delta=21.6\left(\mathrm{CH}_{3}, \mathrm{C}-15\right), 67.4(\mathrm{CHOH}$, C-14), 112.1 (CH, C-8), 116.6 (CH, C-4), 120.6 (CH, C-6),
121.7 (C, C-12), 122.0 (CH, C-5), 128.3 (CH, C-7), 128.5 (C, C-11), 135.8 (C, C-10), 136.6 (C, C-13), 140.7 (C, C-3), 147.4 (C, C-1), 167.1 (C, C-16).

\section{RESULTS AND DISCUSSION}

L-tryptophan and L-lactic acid are commercially available as natural products. Firstly, in order to improve the reactivity of amino group, L-tryptophan methyl ester is obtained via esterification of L-tryptophan ${ }^{17}$. Compound 3 can be obtained through $N$-acylation reaction with L-tryptophan methyl ester, L-lactic acid or L-lactic acid methyl ester. But, the yield of product is low and purification difficult. So, (S)-2-acetoxypropionyl chloride was used instead of L-lactic acid methyl ester or L-lactic acid. What was more, -NH- on compound 2 did not need to be protected because (S)-2-acetoxypropionyl chloride tended to react with $-\mathrm{NH}_{2}$ much more easily. Due to the much quicker rate of anhydride hydrolyzation than the one of ester hydrolyzatoin in compound $\mathbf{8}$, compound $\mathbf{9}$ could be obentained by controlling the quantization of water. The obtained crude product compound $\mathbf{1 0}$ was not purified and used directly.

Compound 4 could be synthesized through BischlerNapieralski reaction ${ }^{18-22}$. But the cyclization reaction under catalysis of $\mathrm{PCl}_{5}, \mathrm{POCl}_{3}$ and $\mathrm{P}_{2} \mathrm{O}_{5}$, gave only compound $\mathrm{A}$ (Fig. 1) which lost the chiral group. Manske ${ }^{23}$ had reported aluminum chloride as cyclizing agent for synthesis of tetrahydroisoquinolines at 1927 . So aluminum chloride was used in our synthesis route as a mild catalyst and target compound 4 was obtained in a very satisfied yield $(87.3 \%$ yield). Dichotomine A is obtained via oxidation reaction and hydrolysis reaction.

\section{Conclusion}

We have achieved a new total synthesis of dichomonine A via a modified Bischler-Napieralski reaction which aluminium chloride is used as a mild cyclization reagent instead of $\mathrm{PCl}_{5}$, $\mathrm{POCl}_{3}$ or $\mathrm{P}_{2} \mathrm{O}_{5}$. Dichomonine A was obtained in 5 steps and in $63.9 \%$ yield. It also provides a novel method to synthesize dichomonines B, C and D.

\section{ACKNOWLEDGEMENTS}

The authors are thankful to the Fundamental Research Funds for the Central Universities for financial support. The item number is lzujbky-2010-137.

\section{REFERENCES}

1. A. Bolognese, G. Correale, M. Manfra, A. Lavecchia, O. Mazzoni, E. Novellino, P. La Colla, G. Sanna and R. Loddo, J. Med. Chem., 47, 849 (2004).

2. M.A. Lynch, O. Duval, A. Sukhanova, J. Devy, S.P. Mackay, R.D. Waigh and I. Nabiev, Bioorg. Med. Chem. Lett., 11, 2643 (2001).

3. J. Ishida, H.-K. Wang, K.F. Bastow, C.-Q. Hu and K.-H. Lee, Bioorg. Med. Chem. Lett., 9, 3319 (1999).

4. J. Ishida, H.K. Wang, M. Oyama, M.L. Cosentino, Q.H. Chang and K.H. Lee, J. Nat. Prod., 64, 958 (2001).

5. M. Laronze, M. Boisbrun, S. Leonce, B. Pfeiffer, P. Renard, O. Lozach, L. Meijer, A. Lansiaux, C. Bailly, J. Sapi and J.-Y. Laronze, Bioorg. Med. Chem., 13, 2263 (2005).

6. A.M. Sobhani and S.A. Ebrahimi, J. Pharm. Sci., 5, 19 (2002).

7. B. Sun, T. Morikawa, H. Matsuda, S. Tewtrakul, L.J. Wu, S. Harima and M. Yoshikawa, J. Nat. Prod., 67, 1464 (2004). 
8. N. Sotomayor, E. Dominguez and E. Lete, J. Org. Chem., 61, 4062 (1996).

9. R.S. Kusurkar, S.K. Goswami and S.M. Vyas, Tetrahedron Lett., 44, 4761 (2003).

10. (a) J.P. Wolfe, in ed.: J.J. Li, Name Reactions in Heterocyclic Chemistry, John Wiley \& Sons, p. 376 (2005); (b) D.D. Holsworth, in ed.: J.J. Li, Name Reactions in Heterocyclic Chemistry, John Wiley \& Sons, p. 465 (2005).

11. M. Agnusdei, M. Bandini, A. Melloni and A. Umani-Ronchi, J. Org. Chem., 68, 7126 (2003).

12. H. Zhang and R.C. Larock, Org. Lett., 3, 3083 (2001).

13. N. Kanekiyo, T. Kuwada, C. Tominari and S. Hibino, J. Org. Chem., 66, 8793 (2001)

14. K. Omura, T. Choshi, S. Watanabe, Y. Satoh, J. Nobuhiro and S. Hibino, Chem. Pharm. Bull., 56, 237 (2008).
15. I. Nemet and L. Varga-Defterdarovic, Bioorg. Med. Chem., 16, 4551 (2008).

16. Q. Zhang, J. Dong, X.-X. Shi and X. Lu, Eur. J. Org. Chem., 17, 3317 (2012).

17. D. Robaa, C. Enzensperger, A.S. Eldin, M.M. Hefnawy, H.I. El-Subbagh, T.A. Wani and J. Lehmann, J. Med. Chem., 54, 7422 (2011).

18. J. Cobo, M. Nogueras, J.N. Low and R. Rodríguez, Tetrahedron Lett., 49, 7271 (2008).

19. E. Awuah and A. Capretta, J. Org. Chem., 75, 5627 (2010).

20. G. Bringmann, T. Gulder, B. Hertlein, Y. Hemberger and F. Meyer, J. Am. Chem. Soc., 132, 1151 (2010).

21. (a) D. Vaccari, P. Davoli, C. Ori, A. Spaggiari and F. Prati, Synlett., 2807 (2008); (b) A. Spaggiari, P. Davoli, L.C. Blaszczak and F. Prati, Synlett., 661 (2005).

22. R.Z. Fu, X.X. Xu, Q. Dang and X. Bai, J. Org. Chem., 70, 10810 (2005).

23. R.H. Manske, Chem. Rev., 30, 145 (1942). 